\title{
Uso da tecnologia como recurso didático no ensino em enfermagem: percepções dos estudantes
}

\author{
Luís Felipe Pissaia \\ Enfermeiro e Mestrando em Ensino \\ Especialização em Gestão e Auditoria dos Serviços da Saúde \\ Universidade do Vale do Taquari - UNIVATES - Brasil \\ 冈1pissaia@universo.univates.br \\ Sabrina Monteiro \\ Mestranda em Ensino \\ Especialização em Administração Escolar, Orientação e Supervisão \\ Universidade do Vale do Taquari - UNIVATES - Brasil \\ Juliana Thomas \\ Enfermeira e Mestranda em Ensino na Saúde \\ Especialização em Docência na Educação Profissional \\ Universidade Federal de Ciências da Saúde de Porto Alegre - Brasil \\ Arlete Eli Kunz da Costa \\ Enfermeira e Doutora em Ambiente e Desenvolvimento \\ Universidade do Vale do Taquari - UNIVATES - Brasil
}

Recebido em 17 de janeiro de 2019

Aceito em 23 de maio de 2019

\section{Resumo:}

Este estudo objetiva verificar as percepções de estudantes de enfermagem sobre o uso de tecnologias digitais em sala de aula em uma Instituição de Ensino Superior do interior do Rio Grande do Sul, Brasil. Trata-se de um estudo descritivo e exploratório, com abordagem qualitativa. Participaram 31 estudantes da disciplina de Exercício Profissional, integrante do currículo específico do curso de graduação em enfermagem. A coleta dos dados ocorreu por meio da aplicação em sala de aula de questionário estruturado, com quatro perguntas sobre o assunto. Para a análise de dados, utilizou-se a Análise de Conteúdo teorizada por Bardin, para tanto, foram elencadas quatro categorias: O professor e suas tecnologias: as percepções dos alunos sobre o uso em sala de aula; Oportunidades do uso de tecnologias em sala de aula: a prática do professor a partir das percepções dos estudantes; Ensino e tecnologias: compreensões dos estudantes sobre sua relação; A formação em enfermagem e o uso de tecnologias: intersecções sobre o mercado de trabalho. Partindo da análise dos dados, é possível inferir que houve dois posicionamentos em relação aos estudantes, aqueles que percebem o uso da tecnologia como algo importante no dia-a-dia e outros que, ao contrário, não a utilizam com tanta profundidade para a sua aprendizagem.

Palavras-chave: Ensino, Ensino em Enfermagem, Tecnologias em Sala de Aula, Tecnologias em Saúde, Ensino em Saúde, Instituição de Ensino Superior. 
Uso da tecnologia como recurso didático no ensino

em enfermagem: percepções dos estudantes

\title{
Use of technology as a teaching resource in nursing teaching: students' perceptions
}

\begin{abstract}
:
This study aims to verify the perceptions of nursing students about the use of digital technologies in the classroom in a Higher Education Institution in the interior of Rio Grande do Sul, Brazil. This is a descriptive and exploratory study with a qualitative approach. Thirty-one students participated in the discipline of Professional Exercise, part of the specific curriculum of the nursing undergraduate course. Data were collected through the classroom application of a structured questionnaire, with four questions about the subject. For the data analysis, we used the Content Analysis theorized by Bardin, for which four categories were listed: The teacher and his technologies: the students' perceptions about the use in the classroom; Opportunities for the use of technologies in the classroom: the teacher's practice based on students' perceptions; Teaching and technologies: students understand their relationship; Training in nursing and the use of technologies: intersections on the labor market. Based on the data analysis, it is possible to infer that there were two positions in relation to the students, those who perceive the use of technology as something important in everyday life and others who, on the contrary, do not use it so deeply for their learning.
\end{abstract}

Keywords: Teaching, Teaching in Nursing, Classroom Technologies, Health Technologies, Health Teaching, Institution of Higher Education.

\section{Uso de la tecnología como recurso didáctico en la enseñanza en enfermería: percepciones de los estudiantes}

\section{Resumen:}

Este estudio tiene como objetivo verificar las percepciones de estudiantes de enfermería sobre el uso de tecnologías digitales en el aula en una Institución de Enseñanza Superior del interior de Rio Grande do Sul, Brasil. Se trata de un estudio descriptivo y exploratorio, con abordaje cualitativo. Participaron 31 estudiantes de la disciplina de Ejercicio Profesional, integrante del currículo específico del curso de graduación en enfermería. La recolección de los datos ocurrió por medio de la aplicación en aula de cuestionario estructurado, con cuatro preguntas sobre el tema. Para el análisis de datos, se utilizó el Análisis de Contenido teorizado por Bardin, para lo cual se incluyeron cuatro categorías: El profesor y sus tecnologías: las percepciones de los alumnos sobre el uso en el aula; Oportunidades del uso de tecnologías en el aula: la práctica del profesor a partir de las percepciones de los estudiantes; Enseñanza y tecnologías: comprensión de los estudiantes sobre su relación; La formación en enfermería y el uso de tecnologías: intersecciones sobre el mercado de trabajo. A partir del análisis de los datos, es posible inferir que hubo dos posicionamientos en relación a los estudiantes, aquellos que perciben el uso de la tecnología como algo importante en el día a día y otros que, por el contrario, no la utilizan con tanta profundidad para su propia el aprendizaje.

Palabras-clave: Educación, Enseñanza en Enfermería, Tecnologías en Sala de classe, Tecnologías en Salud, Enseñanza en Salud Institución de enseñanza superior. 


\section{INTRODUÇÃO}

O ensino de determinado conceito ou fórmula necessita do empenho, tanto por parte do estudante, como do docente, uma vez que estes estruturam pensamentos e conjecturas sobre a resolução de determinado problema que de fato caracterizem a compreensão e a significância da situação. Neste sentido, o aprender em enfermagem se torna distinto, já que, conforme Pereira e Cardoso (2017), a área exige muito mais do que aulas teóricas, requer experiências reais de vida, pois o significado de cada modelo teórico experimentado em sala de aula necessita de vivências que ofereçam um momento de reflexão e construção de hipóteses ao estudante.

Em seu estudo, Gilbertoni et al. (2018) reforçam tal afirmação, inferindo sobre a necessidade de introduzir o ensino ao contexto socioambiental em que o estudante se encontra. Deste modo, o estudante poderá construir o conhecimento sob pressupostos práticos, ou seja, por meio do "saber fazer" ou da "tentativa de fazer", experimentando na realidade os processos assimilados durante sua trajetória.

Os pressupostos de uma educação prática, como citado por Gomes Lima et al. (2017), está no meio em que o ensino ocorre, ou seja, por mais que frequentemente os momentos teóricos sejam necessários, a experimentação em campo, a testagem dos modelos e inferências sobre o cotidiano devem acontecer, com base em recorrências dos diversos conteúdos assimilados e construídos durante a realização do curso. Acrescentam-se, também, os pressupostos da enfermagem moderna que usufrui do embasamento prático por representar a necessidade de seus estudantes construírem uma aprendizagem significativa.

Neste sentido, Riegel e Crossetti (2017) enfatizam as múltiplas faces da formação do enfermeiro, possuindo, além do ensino teórico e prático, as atividades de pesquisa e extensão que favorecem sua correlação e esta, por sua vez, torna-se o princípio da compreensão e construção da relação entre teoria e prática, haja vista os recursos utilizados no decorrer das aulas.

Segundo Maffissoni, Vendruscolo e Lima Trindade (2017), as demandas políticas e econômicas são impactantes, por definir os campos de atuação do futuro enfermeiro, direcionando a realização de práticas assistências e exigências legais. Acompanhando essas mudanças, os currículos das escolas de enfermagem foram gradualmente sendo modificados e incorporaram necessidades tecnológicas antes não contempladas nos currículos 
especializados, introduzindo novos modelos de atuação, conforme comentam Santos et al. (2017).

$\mathrm{Na}$ contemporaneidade, o perfil do enfermeiro contempla, principalmente, as áreas de administração e gestão dos espaços de saúde, áreas estas que utilizam instrumentos específicos da profissão para auxiliar nestes processos (GOMES LIMA et al.., 2017). No entanto, tal demanda exige capacitação dos estudantes, por meio de experiências em campo, de toda a aprendizagem ocorrida em sala de aula (SANTOS et al.., 2017).

Para tanto, as diretrizes curriculares para os cursos de enfermagem, preconizadas pelo Conselho Nacional de Educação (CNE) (2001) aludem que as Instituições de Ensino Superior (IES) promovam a relação entre teoria e prática para seus estudantes, buscando uma formação qualificada e comprometida com a realidade locorregional. Dessa forma, a relação entre teoria e prática compõe uma das principais exigências para a formação do enfermeiro, sendo descrita por Gomes Lima et al. (2017) como uma experiência intrínseca própria de cada estudante, por despertar individualmente as conjecturas necessárias para a compreensão do conteúdo teórico em sala de aula e sua efetiva realização no campo prático.

Sendo assim, o acadêmico se torna-capaz de refletir e correlacionar ambos os momentos em sua trajetória (GOMES LIMA et al.., 2017). Ainda na opinião de Gomes Lima et al. (2017), o estudante utiliza-se da argumentação para compreender e articular métodos de ensino capazes de sintetizar e oferecer uma visão ampla dos conteúdos ao estudante. Em outras palavras, Santos et al. (2017) explicitam a utilização de recursos que possam estar presentes na rotina acadêmica com a finalidade de integrar a teoria com a prática, instrumentalizando um "saber fazer" efetivo e condizente com as habilidades e competências esperadas do enfermeiro.

Em adição, Souza, Colliselli e Madureira (2017) reafirmam a importância da formação em enfermagem generalista, holística e reflexiva, baseada nos preceitos éticos e morais e comprometidos com as mudanças sociais e ambientais e que estejam engajados com seu compromisso cidadão. Neste contexto Pissaia et al. (2018) introduzem à discussão a necessidade de inserção das tecnologias digitais em sala de aula, as quais se tornam importantes ao atual desenvolvimento do ensino em enfermagem. 
Deste modo, as tecnologias digitais assumem um papel de inovação e qualificação do ensino em enfermagem, inserindo um novo campo de atuação e desenvolvimento de práticas na área, conforme comenta Pissaia et al. (2018). Desta forma, torna-se importante conhecer as percepções de estudantes de enfermagem sobre o uso de tecnologias digitais em sala de aula, sendo que as mesmas representam um diferencial em seus processos de ensino e aprendizagem. Neste contexto, o objetivo deste estudo é verificar as percepções de estudantes de enfermagem sobre o uso de tecnologias digitais em sala de aula em uma IES do interior do Rio Grande do Sul, Brasil.

\section{METODOLOGIA}

Trata-se de um estudo descritivo e exploratório, com abordagem qualitativa. Os participantes da pesquisa foram 31 estudantes do curso de graduação em enfermagem de uma IES do interior do Rio Grande do Sul, Brasil.

Delimitaram-se os participantes a partir de sua matrícula na disciplina de Exercício Profissional, a qual se faz presente do currículo específico do presente curso de graduação. A pesquisa foi realizada após a introdução do conteúdo "Ensino e Tecnologias", a qual instigou o docente a buscar saber a percepção de seus próprios alunos.

O critério de inclusão foi todos os estudantes regularmente matriculados na disciplina durante o semestre citado. Os alunos foram convidados pelo docente a participar do estudo, sendo explicado o objetivo da pesquisa e os critérios éticos que envolvem todo o processo, foi entregue o Termo de Consentimento Livre e Esclarecido (TCLE), o qual foi lido e assinado em duas vias de igual teor, uma ficou em posse do pesquisador e outra dos estudantes.

A disciplina contava com 31 estudantes regularmente matriculados, sendo que todos aceitaram participar. A coleta dos dados ocorreu por meio da aplicação em sala de aula de questionário estruturado com quatro perguntas sobre o assunto. Os estudantes tiveram o tempo delimitado de duas horas e trinta minutos para devolver o questionário ao pesquisador. 
Após receber os questionários, os resultados passaram pela pré-análise onde foram compilados e divididos por pontos focais, os quais foram representados por categorias temáticas, sendo analisados e discutidos com o apoio bibliográfico pertinente, seguindo aproximações com a Análise de Conteúdo de Bardin (2016). Para apresentação dos resultados, utilizaram-se codinomes para nomear os participantes, sendo identificados pela letra "A" maiúscula, seguida de números ordinais sorteados aleatoriamente.

Todo o processo de pesquisa foi norteado pelos preceitos éticos previstos pela Resolução 466/12 sobre estudos realizados com seres humanos. A realização deste estudo justifica-se pela necessidade de compreender a relação entre as tecnologias digitais e a formação do profissional de enfermagem, complementando o projeto de pesquisa "Impacto das tecnologias da informática no processo de Sistematização da Assistência de enfermagem em uma instituição hospitalar do Vale do Taquari/RS, Brasil”, aprovado pelo Comitê de Ética em Pesquisa da Universidade do Vale do Taquari por meio do Parecer nº 1.379.976.

\section{RESULTADOS E DISCUSSÃO}

Nesta seção disserta-se sobre os resultados alcançados através da intervenção realizada. Para tanto, com aproximações da Análise de Conteúdo de Bardin (2016), elencouse quatro categorias, que serão descritas a seguir. As quais foram nomeadas: "O professor e suas tecnologias: as percepções dos alunos sobre o uso em sala de aula", "Oportunidades do uso de tecnologias em sala de aula: a prática do professor a partir das percepções de seus estudantes", "Ensino e tecnologias: compreensões dos estudantes sobre sua relação", e "A formação em enfermagem e o uso de tecnologias: intersecções sobre o mercado de trabalho".

O professor e suas tecnologias: as percepções dos alunos sobre o uso em sala de aula

Nesta categoria, elencaram-se as percepções dos participantes sobre o uso de tecnologias em sala de aula pelo professor. Neste aspecto surgem questões pertinentes à 
percepção do aluno frente às tecnologias, sendo possível questionar sobre o que é tecnologia digital e os reflexos da sua utilização na visão do discente, como é possível observar a seguir:

Considero que muito eficaz, um bom exemplo de tecnologia é os slides, que de alguma forma ajudam a passar o conteúdo, principalmente quando o tema é bem complexo e a turma precisa de reforço. É legal que dá para ter imagens e estudar depois (A2).

Observa-se um modelo de ensino, ainda que utilizando recursos tecnológicos, com características tradicionais, diante de uma aula expositiva. Para Nóvoa (2015), a pedagogia tradicional é constituída na transmissão dos conhecimentos do professor para os seus alunos. Ainda que existam reflexões e discussões que visam o incentivo a mudanças nas concepções pedagógicas e curriculares, nota-se como um dos dificultadores do processo de adoção de estratégias de ensino em sala o perfil dos alunos, habituados no modelo de ensino tradicional, onde o professor era o detentor do conhecimento.

Para tanto, o uso da tecnologia em sala de aula perpassam os equipamentos utilizados pelo professor, conforme as enunciações abaixo:

Normalmente usam computador, datashow e televisão nas aulas que eu participo. Essas ferramentas sem dúvida ajudam tanto o professor quanto nós a entender o conteúdo e depois estudar, ainda facilita, pois é impossível guardar tudo na memória (A3)

Acho muito importante o uso na sala de aula. Infelizmente nem todos professores usam, isso deve depender de cada um, que organiza as aulas antes do semestre. Mas gosto muito daqueles que usam, pois entendo mais o conteúdo (A5)

Coadunando a concepção positiva apresentada, obtida com essa ferramenta em sala de aula, Delors (2003), enfatiza que a educação é desenvolvida quando o aluno se organiza em torno de quatro aprendizagens, que ao longo da vida serão os pilares do conhecimento: aprender a conhecer, aprender a ser, aprender a viver juntos, aprender a fazer. Deste modo, a tecnologia de ensino anunciado pelo aluno, pode não ser o bastante para que o aprendizado de modo reflexivo de fato aconteça. Importante destacar a frase "guardar tudo na memória", proferida pelo estudante A5, isto retrata um olhar fragmentado, superficial e memorialístico, reforçando o sistema de ensino tradicionalista. 
A educação é um processo ativo, que interage no cotidiano do mundo social, portanto, é indispensável ações que provoquem mudanças e transformações sociais nos assuntos abordados durante o período acadêmico. Corroborando com esta ideia, Pimenta e Anastasiou (2008) destacam que o ensino na universidade pressupõe a busca constante por investigações científicas que comprovem a existência de boas práticas, disseminando o conhecimento.

Refletindo sobre as constantes mudanças e transformações em decorrência da tecnologia em sala de aula, os participantes ressaltaram que:

Percebo que na enfermagem a maioria dos professores usam [as tecnologias], até nas práticas isso tem, pois é o mais atual hoje. Facilita muito tanto nos trabalhos, quando tem que fazer na sala, quanto em casa (A8).

As tecnologias sempre estão na sala de aula, sempre tem notebook na mesa ajudando. Gostei muito de uma atividade em que a turma produziu vídeos na própria sala, isso foi legal e o professor ensinou a fazer (A11).

O uso de tecnologias é muito bom, porque facilita nas atividades, conseguimos fazer pesquisas e ver vídeos sobre o assunto trabalhado em aula (A17).

Agregando às considerações dos estudantes, Masetto (2006, p. 144) destaca que, "haverá necessidade de variar estratégias tanto para motivar o aprendiz como para responder aos diferentes ritmos e formas de aprendizagem, pois nem todos aprendem do mesmo modo e no mesmo tempo". o mesmo autor ainda complementa que as tecnologias utilizadas em sala de aula devem valorizar a aprendizagem, incentivar a formação permanente, auxiliar na busca de novas informações, favorecer o debate, a reflexão, a construção de artigos e textos.

\section{Oportunidades do uso de tecnologias em sala de aula: a prática do professor a partir das percepções de seus estudantes}

Nesta seção, os alunos foram questionados sobre as oportunidades nas quais onde o professor poderia utilizar as tecnologias em sala de aula. Dessa forma:

Acredito que sempre há como melhorar e inovar em algo, mas no momento não vejo nenhuma forma (A1). 
Quanto mais tecnologia, mais distração os alunos têm, se for de forma didática até acredito que o uso do projetor seja suficiente (A3).

Por meio destes relatos, é possível inferir que nem sempre o uso de tecnologia é visto como algo que vem a agregar ao processo de ensino e aprendizagem em sala de aula. Quanto a esse aspecto Pérez Gómez (2015) atenta que é imprescindível que o professor tenha objetivos claros com sua prática, por conseguinte o uso por si só pode se tornar algo irrelevante neste processo. No entanto, esta ferramenta pode ser uma aliada para motivar e incentivar o discente a melhorar o seu desempenho durante o período escolar.

O uso da tecnologia, como proposta educadora, minimiza a distração, e promove a integração do aluno com o contexto proposto pelo docente. Cabe ressaltar que a prática e exploração dos recursos tecnológicos desviam da metodologia tradicional de ensino, e cabe ao professor elaborar e definir objetivos e regras para sua utilização, a fim de evitar a utilização inadequada devido às facilidades técnicas de uso, em detrimento dos potenciais educativos. Em consonância:

Diferentes tecnologias poderiam ser usadas em pesquisas na sala de aula. Poderiam ser usados os celulares, o computador, fazendo pesquisas diferentes com esses aparelhos (A4).

Haveria sim, em trabalhos em grupos sobre assuntos diferentes, por algo que nos chame a atenção para que possamos ir além do que apenas pesquisar em casa sobre o tema (A7).

De acordo com Coll (2000), existem dois motivos para estimular o discente ao aprendizado: uma delas está relacionada ao assunto abordado em sala, enquanto o outro envolve a atitude do docente, haja vista os novos elementos aos já existentes em suas habilidades cognitivas. Em consonância, almeja-se inserir cada vez mais a utilização de estratégias que visem a interação e estimulem a motivação dos discentes, tornando-os profissionais críticos, habilitados a resolução de problemáticas e situações de eventualidades do dia a dia.

Utilizar recursos que melhorem a atitude do aluno em sala de aula, colaboram para que aconteça uma aprendizagem mais significativa. Porém, deve-se atentar para a forma como isso correrá: 
Gosto da maneira como está, prefiro mais trabalhos feitos a mão do que simplesmente Ctrl + c e Ctrl + v na internet (A10).

Com isso, ratifica-se a concepção de Pérez Gómez (2015) que evidencia em seus estudos o uso da tecnologia na atualidade. Para ele, o professor não pode simplesmente ignorar a "era digital" (PÉREZ GÓMEZ, 2015, p. 10) na qual a sociedade encontra-se inserida, porém deve atentar para o seu uso adequado que contemple o processo de ensino e aprendizagem de maneira significativa, não apenas de mera transmissão de conhecimentos.

Neste mesmo sentido, Chirelli (2002), afirma que o currículo de enfermagem deve abordar, valorizar e articular o ensino, serviço e comunidade no processo ensino aprendizagem estimulando o aluno a refletir sobre as suas ações na realidade onde está inserido, buscando solucionar problemas do cotidiano e utilizando a aprendizagem já obtida como propulsora para formação de uma aprendizagem crítica e reflexiva. Conforme pode ser visto nos relatos abaixo:

Todos os alunos poderiam ter acesso ao notebook ou algo do tipo para não precisar imprimir os slides e os artigos (A11).

Poderia ser de forma mais frequente com a finalidade de adquirir outros pensamentos e conceitos sobre o assunto e não só a parte teórica (A14).

Como a maioria hoje tem celular com acesso a internet, seria mais interessante fazer questões online. Tive aula onde o professor elaborou questões para responder depois do conteúdo passado e todos aprenderam o conteúdo (A18).

Partindo destes pressupostos, são perceptíveis que os recursos que vão além do espaço escolar, são tarefas que demandam atenção, limites e que pode proporcionar a valorização da aprendizagem significativa, preconizada por Ausubel (2000). Para este aspecto é preciso que o aluno tenha maturidade para saber lidar com os recursos que estão disponíveis em seu meio.

\section{Ensino e tecnologias: compreensões dos estudantes sobre sua relação}

Nesta categoria, os estudantes foram instigados a descrever sua compreensão sobre a relação entre ensino em saúde e tecnologias. Dessa forma: 
Que o ensino em saúde se torna mais fácil com o uso das tecnologias, se forem usadas de maneira apropriada, para que ajude a nós aprendermos (A2).

Assim sendo, compete à instituição proporcionar meios eficazes aos alunos para que estimule a construção do conhecimento e adquira competências, habilidades e atitudes, por isso:

Acredito que a tecnologia abrange grande parte do conhecimento existente. A saúde só tem a crescer com o auxílio das tecnologias, cada vez aprimorando e avançando no mundo da saúde, sendo na indústria farmacêutica ou em sistemas dentro do hospital (A4).

Por vezes, os professores que dispõe de realizar atividades com o uso do computador, fazem na maioria das vezes, de forma tradicional. Tornando as aulas monótonas e sem aprendizagem significativa, onde o conteúdo é apenas transmitido, de forma acumulativa. Desta forma, os alunos se sentem desmotivados e dispersos (CAMPOS, 2007), uma vez que:

Não adianta sabermos tecnologias sem saber o que realmente um enfermeiro faz, até porque o computador não consegue saber o que o paciente tem (A7).

Nessa acepção, Almeida (2001, p.3) lembra que usar as tecnologias na educação é mais que explorar recursos computacionais ou navegar na Internet: seu uso deve estar "voltado à promoção da aprendizagem". Ademais, o professor exerce uma importante influência no êxito do processo de ensino aprendizado. É com base nas escolhas de atividades, teorias, estratégias de ensino que o enfermeiro professor pode atingir a atenção e interesse do aluno, ampliando assim as chances de ocorrer o aprendizado (BASTABLE, 2013). Uma vez que:

Nos últimos anos a tecnologia está crescendo e ajudando muito na área da saúde, principalmente em procedimentos, questões de organização de informações (A9).

As novas tecnologias facilitam muito as coisas, mas vai de cada pessoa se quer aprender ou não, basta partir do interesse dela (A10).

Assim sendo, o processo de ensino aprendizagem na saúde, quando estimulado no modo crítico entre professor e aluno, pode favorecer o conhecimento profissional voltado à reflexão sobre a prática em si. Para que ocorra em sala de aula uma sintonia entre professor e aluno, o docente precisa estar atento às singularidades desses alunos, seu universo cognitivo e cultural (NOVOA, 1997). 
Nesta mesma perspectiva, Moran (2006, p. 36) destaca que “[...] a educação escolar precisa compreender e incorporar mais as novas linguagens, desvendar os seus códigos, dominar as possibilidades de expressão e as possíveis manipulações”. Dessa forma, é importante educar para usos democráticos, mais progressistas e participativos das tecnologias, que facilitem a evolução dos indivíduos.

\section{A formação em enfermagem e o uso de tecnologias: intersecções sobre o mercado de trabalho}

Nesta categoria os estudantes dissertaram sobre as demandas no uso de tecnologias no mercado de trabalho, a partir da formação atual. Tal concepção levou a salientarem:

Considero que sim, usamos as tecnologias, no entanto sempre tem que seguir buscando novidades sobre a área para não desatualizar (A3).

Sempre devemos procurar por mais conhecimento, seja na área da saúde ou na tecnologia, nunca saberemos tudo, mas podemos nos aperfeiçoar (A7).

A tecnologia auxilia no meu aprendizado e no crescimento do conhecimento (A12).

Às vezes acho que utilizo demais a tecnologia perante a enfermagem, principalmente o celular em sala de aula (A9).

Partindo do evidenciado, considera-se a importância da busca constante de informações e aperfeiçoamento na área em destaque. Pérez Goméz (2015) ratifica que a formação contínua do cidadão contemporâneo exige provocar o desenvolvimento da capacidade de trabalhar criativa e colaborativamente em equipes, criar contextos sociais de apoio, confiança e colaboração, compreender e estimular as diferenças, lidar com as discrepâncias e desenvolver competências e interação cultural e social, liderança e iniciativa. o que implica diretamente no aprendizado contínuo, conforme a concepção apresentada.

Para Pérez Gómez (2015, p. 141) alude que “[...] ajudar a se educar é o objetivo e a tarefa central do docente na era digital". Dessa forma, assumindo a filosofia pedagógica de que os alunos devem ser os geradores de seu próprio conhecimento e os professores os facilitadores deste processo, abrem-se as múltiplas possibilidades metodológicas que devem estar permanentemente disponíveis para o docente. 
Porém, pensando de forma inusitada em meio aos relatos aqui apresentados, um dos alunos enfatiza que:

Considero a minha utilização não produtiva, pois não utilizo a tecnologia para me informar de assuntos didáticos, fico só conversando com outras pessoas (A15)

Pressupondo que o uso da tecnologia não ser essencial na prática diária de referida aluna, Almeida (2005) destaca que "Criar espaços para a identificação e o diálogo entre essas formas de linguagem e permitir que os alunos se expressem de diferentes maneiras são ações que favorecem o desenvolvimento da consciência crítica". Dessa forma, pensando que a tecnologia está à disposição para auxiliar e agilizar muitos procedimentos, Pérez Gómez (2015) enfatiza que não se pode deixar de levar esse fator em consideração, ressaltando seu uso consciente.

\section{CONSIDERAÇÕES FINAIS}

Ao findar a pesquisa aqui enunciada, pode-se destacar que, conforme as percepções da maioria dos estudantes de enfermagem, o uso das tecnologias pode auxiliar o ensino, porém deve ter objetivos claros, para que não se torne algo insignificante. O uso por si só; pode não resultar em aspectos que tenham êxito, levando em consideração também as concepções e percepções que os estudantes apresentam por meio de seus relatos.

Em contrapartida, alguns estudantes evidenciaram que preferem aulas práticas, destacando que "o computador não consegue saber o que o paciente tem", acredita-se que isso pode ser resultado de experiências que foram irrelevantes quanto ao uso da tecnologia. Percebe-se que este grupo de participantes não conseguem por vezes, compreender que a tecnologia pode ser aliada aos conhecimentos práticos, utilizados principalmente no cotidiano dos enfermeiros, uma vez que identificam como uma mera transmissão de conhecimentos, sem aplicabilidade, tornando as aulas expositivas sem interações com a realidade. 
Este estudo apresenta as percepções de uma parcela dos estudantes de enfermagem da IES, por este motivo, os pesquisadores motivam-se a buscar novas pesquisas e ampliar o conhecimento sobre as próprias práticas e uso de tecnologias em sala de aula. Acredita-se que estudos como este, qualificam as práticas docentes e compõem a estrutura científica necessária para a mudança dos padrões de ensino, principalmente, fazendo com que os estudantes reflitam sobre o seu próprio ensino, sendo uma dificuldade enfrentada neste estudo. Ao fim, ressalta-se que em combinação prévia com os estudantes, os resultados oriundos da pesquisa serão apresentados aos seus participantes por meio de trabalhos científicos.

\section{REFERÊNCIAS}

ALMEIDA, M. E. B. Prática e formação de professores na integração de mídias. Prática pedagógica e formação de professores com projetos: articulação entre conhecimentos, tecnologias e mídias. In: ALMEIDA, M. E. B.; MORAN, J. M. (Orgs.). Integração das tecnologias na educação. Brasília,DF: MEC/SEED, 2005. p.39-45

AUSUBEL, D. P. The Yacquisition and retention of knowledge: a cognitive view. Dordrecht: Kluwer Academic Publisher, 2000. 210p.

BARDIN, L. Análise de Conteúdo. Tradução: Luís Augusto Pinheiro. São Paulo: Edições 70, 2016.

BASTABLE, S. B. O Enfermeiro como Educador: princípios de ensino aprendizagem para a prática de enfermagem - 3.ed. Porto Alegre: Artmed, 2010. 688p.

CAMPOS, P. K. A formação docente integrada ao ambiente computacional e sua (re)signifi cação na prática pedagógica em matemática: análise de um caso. 2007. 152f. Dissertação (Mestrado em Educação) Universidade São Francisco, Itatiba, SP, 2007.

CHIRELLI. M. Q. O processo de formação do enfermeiro crítico-reflexivo na visão dos alunos do curso de enfermagem da FAMEMA. Tese (Doutorado em Enfermagem). Ribeirão Preto(SP): Escola de Enfermagem de Ribeirão Preto, Universidade de São Paulo ;2002. 286 f.

COLL, C. Psicologia e currículo: uma aproximação psicopedagógica a elaboração do currículo escolar. São Paulo: Ática; 2000

CONSELHO NACIONAL DE EDUCAÇÃO. Câmara de Educação Superior. Resolução CNE/CES N. 3, de 07 de novembro de 2001. Institui as Diretrizes Curriculares Nacionais do curso de graduação em enfermagem. Diário Oficial da República Federativa da União. Brasília, 09 nov. 2001. Seção 1, p. 37.

DELORS, J. Educação: um tesouro a descobrir. 8. Ed. São Paulo: Cortez; Brasília, DF: MEC: UNESCO, 2003. FREITAS, R. C. Diálogos possíveis: os desafios do uso da internet na escola. 2006. 95f. Dissertação (Mestrado em Educação) Universidade Federal do Paraná, Curitiba, 2006.

GIBERTONI, J. et al.. Plano de ensino de enfermagem em centro cirúrgico. Revista da Escola de Enfermagem da USP, v. 7, n. 2, p. 152-176, 2018. 
GOMES LIMA, M. F. et al.. Desenvolvendo competências no ensino em enfermagem obstétrica: aproximações entre teoria e prática. Revista Brasileira de Enfermagem, v. 70, n. 5, 2017.

MAFFISSONI, A. L.; VENDRUSCOLO, C.; LIMA TRINDADE, L. Redes de atenção à saúde no VER-SUS Oeste Catarinense: contribuições para o ensino em enfermagem. Revista de Enfermagem da UFSM, v. 7, n. 2, p. 167178, 2017.

MASETTO. M. T. Mediação pedagógica e o uso da tecnologia. In: MORAN, J. M.; MASETTO, M. T.; BEHRENS, M. A. Novas tecnologias e mediação pedagógica. 8. ed. Campinas, SP: Papirus, 2006. p.133-173.

MORAES, S. A. O uso da internet na prática docente: reflexões de uma pesquisadora em ação. 2006. 107f. Dissertação (Mestrado em Educação) Universidade Estadual de Maringá, Maringá, 2006.

MORAN, J. M. Ensino e aprendizagem inovadores com tecnologias audiovisuais e telemáticas. In: MORAN, J. M.; MASETTO, M. T.; BEHRENS, M. A. Novas tecnologias e mediação pedagógica. 12. ed. Campinas,SP: Papirus. 2006. p.11-66.

NÓVOA, A. Carta a um jovem investigador em educação. Investigar em Educação, Porto, n. 3, p. 13-21, 2015.

PEREIRA, L. G. M.; CARDOSO, A. L. A formação profissional do enfermeiro docente, que atua no ensino técnico: e o saber formar profissionais capazes de pensar e gestar soluções. Revista Uningá, v. 54, n. 1, 2017.

PÉREZ GÓMEZ, A. L. Educação na era digital: a escola educativa. Porto Alegre: Penso, 2015. 192p.

PIMENTA, S. G.; ANASTASIOU, L. G. C. Docência no Ensino Superior. 2. ed. São Paulo, SP: Cortez, 2005.

PISSAIA, L. F. et al.. Impacto de tecnologias na implementação da sistematização da assistência de enfermagem hospitalar: uma revisão integrativa. Revista de Epidemiologia e Controle de Infecção, v. 1, p. 1-20, 2018.

RIEGEL, F,; CROSSETTI, M. G. O. Pensamento crítico holístico no ensino da enfermagem. Simpósio do Processo de Enfermagem (8.: 2017: Porto Alegre, RS) Processo de enfermagem: estratégia para resultados seguros na prática clínica. Porto Alegre: HCPA, 2017.

SANTOS, E. O. et al.. Aprendizagem baseada em problemas no ensino da enfermagem. Revista Contexto \& Saúde, v. 17, n. 32, p. 55-66, 2017.

SOUZA, J. B.; COLLISELLI, L.; MADUREIRA, V. S. F. A utilização do lúdico como estratégia de inovação no ensino da enfermagem. Revista de Enfermagem do Centro-Oeste Mineiro, v. 7, 2017.

(cc) EY

Este trabalho está licenciado com uma Licença Creative Commons - Atribuição 4.0 Internacional. 